\title{
Clinical Significance of the Interval Change of Plasma Neutrophil Gelatinase-Associated Lipocalin in Acute Kidney Injury and Acute Kidney Injury Superimposed on Chronic Kidney Disease
}

\author{
Ha Yeon Kim, Chang Seong Kim, Eun Hui Bae, Soo Wan Kim, and Seong Kwon Ma* \\ Department of Internal Medicine, Chonnam National University Medical School, Gwangju, Korea
}

Early detection of acute kidney injury (AKI) is crucial for patients with chronic kidney disease (CKD) because the staging of AKI is associated with in-hospital mortality and CKD is a risk factor for the development of AKI. ${ }^{1}$ The definition of $\mathrm{AKI}$ is primarily based on serum creatinine $(\mathrm{sCr})$ as a surrogate marker for glomerular filtration rate (GFR). However, sCr level may not reflect an acute decline of GFR, and it may be affected by several factors including age, sex, muscle mass, and chronic illness. ${ }^{2}$ The present study investigated the clinical significance of plasma neutrophil gelatinase-associated lipocalin (pNGAL) as a biomarker in the diagnosis of AKI and AKI superimposed on CKD. This retrospective observational study included 343 patients with AKI. The patients were classified into two groups; AKI $(n=90)$ and AKI superimposed on CKD $(n=253)$. AKI and CKD were defined by the 2012 Kidney Disease: Improving
Global Outcomes Clinical Practice Guideline. The cut-off value of pNGAL was $200 \mathrm{ng} / \mathrm{mL}$. The levels of $\mathrm{sCr}$ and pNGAL obtained at baseline and at $48 \mathrm{hr}$ after the onset of AKI were analyzed. The interval change of $\mathrm{sCr}$, estimated GFR (eGFR) and pNGAL during the first $48 \mathrm{hr}$ (a value at baseline-a value at $48 \mathrm{hr}$ ) was described as $\Delta \mathrm{sCr}$, $\triangle \mathrm{eGFR}$ and $\triangle \mathrm{pNGAL}$, respectively. In the present study, the level of pNGAL was inversely correlated with eGFR calculated by the CKD-EPI equation $(r=-0.376, \mathrm{p}<0.001)$, and positively correlated with $\mathrm{SCr}(r=0.421, \mathrm{p}<0.001)$. The levels of sCr at baseline and at $48 \mathrm{hr}$ were higher, and those of eGFR were lower in the AKI superimposed on CKD group than the AKI group. In addition, pNGAL levels at baseline and at $48 \mathrm{hr}$ were higher in the AKI superimposed on CKD group as compared with the AKI group. However, the value of the $\triangle$ pNGAL was higher in the AKI group as compared

TABLE 1. Clinical characteristics of the enrolled patients

\begin{tabular}{lccc}
\hline & AKI $(\mathrm{n}=90)$ & AKI on CKD $(\mathrm{n}=253)$ & p-value \\
\hline Age (years) & $57 \pm 17$ & $61 \pm 16$ & 0.028 \\
Male sex, $\mathrm{n}(\%)$ & $59(65.6)$ & $161(63.6)$ & 0.744 \\
Diabetes, $\mathrm{n}(\%)$ & $25(36.2)$ & $86(52.8)$ & 0.002 \\
sCr at baseline $(\mathrm{mg} / \mathrm{dL})$ & $3.3 \pm 2.9$ & $6.0 \pm 3.4$ & $<0.001$ \\
sCr at $48 \mathrm{hr}(\mathrm{mg} / \mathrm{dL})$ & $2.2 \pm 1.8$ & $4.8 \pm 2.8$ & $<0.001$ \\
$\Delta$ sCr $(\mathrm{mg} / \mathrm{dL})$ & $1.0 \pm 2.4$ & $1.1 \pm 2.0$ & 0.738 \\
eGFR at baseline $\left(\mathrm{mL} / \mathrm{min} / 1.73 \mathrm{~m}^{2}\right)$ & $49.5 \pm 74.4$ & $12.8 \pm 11.4$ & $<0.001$ \\
eGFR at $48 \mathrm{hr}\left(\mathrm{mL} / \mathrm{min} / 1.73 \mathrm{~m}^{2}\right)$ & $61.9 \pm 60.3$ & $17.1 \pm 13.6$ & $<0.001$ \\
$\Delta$ eGFR $\left(\mathrm{mL} / \mathrm{min} / 1.73 \mathrm{~m}{ }^{2}\right)$ & $-12.4 \pm 54.2$ & $87.1 \pm 7.9$ & 0.153 \\
pNGAL at baseline $(\mathrm{ng} / \mathrm{mL})$ & $556.9 \pm 449.9$ & $780.8 \pm 386.1$ & $<0.001$ \\
pNGAL at $48 \mathrm{hr}(\mathrm{ng} / \mathrm{mL})$ & $394.7 \pm 373.0$ & $10.1 \pm 229.7$ & $<0.001$ \\
$\Delta$ pNGAL $(\mathrm{ng} / \mathrm{mL})$ & $159.5 \pm 261.5$ & 0.001 \\
\hline
\end{tabular}

Values are expressed as mean \pm standard deviation of the mean. AKI: acute kidney injury, CKD: chronic kidney disease, sCr: serum creatinine, eGFR: estimated glomerular filtration rate, pNGAL: plasma neutrophil gelatinase-associated lipocalin. The interval change of sCr, eGFR and pNGAL during the first $48 \mathrm{hr}$ (a value at baseline-a value at $48 \mathrm{hr}$ ) was described as $\Delta \mathrm{sCr}, \Delta \mathrm{eGFR}$ and $\Delta \mathrm{pNGAL}$, respectively.

\section{Corresponding Author:}

Seong Kwon Ma

Department of Internal Medicine, Chonnam National University Medical School, 42 Jebong-ro, Dong-gu, Gwangju 61469, Korea

Tel: +82-62-220-6579, Fax: +82-62-225-8578, E-mail: drmsk@jnu.ac.kr
Article History:

Received December 31, 2018

Revised January 9, 2019

Accepted January 15, 2019 
with the AKI superimposed on CKD group (159.5 \pm 261.5 in the AKI group vs. $10.1 \pm 229.7$ in the $\mathrm{AKI}$ on $\mathrm{CKD}$ group, $\mathrm{p}=0.001$ ) although the values of the $\Delta \mathrm{sCr}$ and $\triangle \mathrm{eGFR}$ did not differ between the both groups (Table 1). Many clinical studies have demonstrated the clinical value of pNGAL for the diagnosis and prognosis of AKI. ${ }^{3}$ However, the clinical significance of pNGAL in differentiating AKI and AKI superimposed on CKD have not yet been established. ${ }^{4}$ Our results suggest that pNGAL may be a useful marker of GFR and the analysis of $\triangle \mathrm{pNGAL}$ may be helpful to differentiate AKI from AKI superimposed on CKD.

\section{ACKNOWLEDGEMENTS}

This study was financially supported by Basic Science Research Program through the National Research Foundation of Korea (NRF) funded by the Ministry of Education (NRF-2014R1A1A2058250).

\section{CONFLICT OF INTEREST STATEMENT}

None declared.

\section{REFERENCES}

1. Hsu CY, Ordoñez JD, Chertow GM, Fan D, McCulloch CE, Go AS. The risk of acute renal failure in patients with chronic kidney disease. Kidney Int 2008;74:101-7.

2. Stevens LA, Levey AS. Measured GFR as a confirmatory test for estimated GFR. J Am Soc Nephrol 2009;20:2305-13.

3. Haase M, Bellomo R, Devarajan P, Schlattmann P, Haase-Fielitz A; NGAL Meta-analysis Investigator Group. Accuracy of neutrophil gelatinase-associated lipocalin (NGAL) in diagnosis and prognosis in acute kidney injury: a systematic review and metaanalysis. Am J Kidney Dis 2009;54:1012-24.

4. Doi K, Urata M, Katagiri D, Inamori M, Murata S, Hisagi M, et al. Plasma neutrophil gelatinase-associated lipocalin in acute kidney injury superimposed on chronic kidney disease after cardiac surgery: a multicenter prospective study. Crit Care 2013;17:R270. 